\title{
Association of PD-1 and PD-L1 Genetic Polymorphyisms with Type 1 Diabetes Susceptibility
}

\author{
Chenyue Qian, ${ }^{1}$ Heming Guo, ${ }^{2}$ Xiaohong Chen, ${ }^{2}$ Aiming Shi, ${ }^{1}$ Sicheng Li, ${ }^{2}$ Xin Wang, ${ }^{3}$ \\ Jie Pan $\left(\mathbb{D},{ }^{1}\right.$ and Chen Fang $\mathbb{B D}^{2}$ \\ ${ }^{1}$ Department of Pharmacy, The Second Affiliated Hospital of Soochow University, Suzhou 215004, China \\ ${ }^{2}$ Department of Endocrinology, The Second Affiliated Hospital of Soochow University, Suzhou 215004, China \\ ${ }^{3}$ Department of Endocrinology, Jiangsu Province Hospital of TCM, 155 Hanzhonglu, Jiangsu Nanjing 210029, China
}

Correspondence should be addressed to Jie Pan; pankypan@163.com and Chen Fang; afa9911@sina.com

Received 11 April 2018; Accepted 15 October 2018; Published 11 November 2018

Academic Editor: Marco Songini

Copyright ( $) 2018$ Chenyue Qian et al. This is an open access article distributed under the Creative Commons Attribution License, which permits unrestricted use, distribution, and reproduction in any medium, provided the original work is properly cited.

\begin{abstract}
Aims. The programmed death- (PD-) 1/PD-1 ligand (PD-L) pathway plays an important role in regulating $\mathrm{T}$ cell activation and maintaining peripheral tolerance. Accumulated studies showed that PD-1/PD-L1 pathway was involved in the development of type 1 diabetes (T1DM). Since the genetic background of type 1 diabetes differs greatly among the different population, we aim to investigate the association of genetic polymorphisms in PD-1 and PD-L1 with T1DM susceptibility in Chinese population. Methods. In total, 166 T1DM patients and 100 healthy controls were enrolled into the study. Genomic DNA was extracted from $4 \mathrm{~mL}$ peripheral blood samples collected from each subject. Genotyping of 8 selected SNPs of PD-1 and PD-L1 was carried out by the pyrosequencing PSQ 24 System using PyroMark Gold reagents (QIAGEN). Results. SNP rs4143815 in PD-L1 was significantly associated with T1DM. People carrying the $\mathrm{C}$ allele of rs4143815 suffering less risk of T1DM and T1DM patients with $\mathrm{G} / \mathrm{G}$ genotype showed higher levels of autoantibody (AAB) positive incidence compared with $\mathrm{C}$ allele carriers. No significant associations were found in other SNPs. Conclusions. Our results indicate that rs4143815 of PD-L1 is significantly associated with T1DM and may serve as a new biomarker to predict the T1DM susceptibility.
\end{abstract}

\section{Introduction}

The increased prevalence of diabetes mellitus is considered one of the greatest public health challenges nowadays. Type 1 diabetes mellitus (T1DM), a polygenic autoimmune disease, is resulted from both genetic and environmental factors [1]. Although T1DM has a lower prevalence compared with type 2 diabetes mellitus (T2DM), it is the most common form of diabetes in childhood and has a greater impact on the quality of living life.

Programmed cell death 1 (PD-1) is an immunoinhibitory factor belonging to the CD28/B7 family. It plays a vital role in regulating $\mathrm{T}$ cell activation and maintaining peripheral tolerance as a core costimulatory molecule $[2,3]$. Recently, PD-1 has been widely studied as an immune checkpoint that is applied to the treatment of numerous advanced cancers [4-6]. Programmed death ligand-1 (PD-L1) has been shown to be overexpressed in many cancers, including gastric cancer [7], esophageal cancer, pancreatic cancer, and other human gastrointestinal tumors [8]. Accumulated studies showed that blockage of the interaction between PD-1 and PD-L1 can help with better prognosis in various malignant tumors $[6,9,10]$. However, autoimmune diabetes has been reported after receiving anti-PD-1 therapy for tumor in both mouse models and human cases [11-13]. Thus, we assume that there may be a connection between PD-1/PD-L1 pathway and autoimmune diabetes.

Increasing studies had been committed to the association with PD-1/PD-L1 and autoimmune disease, including systemic lupus erythematosus, ankylosing spondylitis, allergic bronchial asthma, and autoimmune diabetes [14-17]. The role of PD-1 in T1DM has been studied using animal models. As a costimulatory molecule that inhibits $\mathrm{T}$ cell proliferation, PD-1 deficiency was shown to increase the risk of T1DM in 
nonobese diabetic (NOD) mice [18]. Studies had shown that low PD-1 might increase $\mathrm{T}$ cell proliferation and activation which lead to the destruction of beta cells, providing a possible mechanism for T1DM. Lower PD-1 expression was proposed to have associations with the development of T1DM in mouse models [19]. PD-L1 recently had been found expressed in the islets of people with type 1 diabetes [20], and we also found that PD-L1 was significantly reduced in the serum of T1DM patients [21]. Since single nucleotide polymorphisms (SNPs) play vital roles in the transcription and translation of genes and have associations with the occurrence and development of diseases, studies had been devoted to the associations between gene polymorphisms with T1DM susceptibility. Existing researches pointed out that PD-1 and PD-L1 SNPs were associated with T1DM susceptibility in different populations [21-23].

However, there is only a few studies focus on the associations between PD-1/PD-L1 gene polymorphisms and T1DM in Chinese population. Therefore, in the present study we investigated 8 selected SNPs of PD-1 and PD-L1 to ascertain whether PD-1 and PD-L1 SNPs influence the T1DM susceptibility.

\section{Materials and Methods}

2.1. Study Population. A total of 166 T1DM patients and 100 healthy controls were recruited for the study (Table 1). Blood samples were collected after overnight fasting from patients at the Endocrinology Department in the Second Affiliated Hospital of Soochow University, Suzhou, China, between 2013 and 2017. All the T1DM patients were diagnosed following the criteria of the American Diabetes Association (reference from Diabetes Care published by ADA). Samples from healthy blood donors, self-report healthy, were chosen as matched controls. Prior to commencing this study, the approval from the Ethics Review Board of the Second Affiliated Hospital of Soochow University was granted.

2.2. Selection of SNPs and Genotyping. Eight SNPs in 2 genes (PD-1 and PD-L1) were selected from the National Center for Biotechnology Information (NCBI) database (Table 2). All of the SNPs satisfied the following criteria: (1) SNPs that are associated with autoimmune disease occurrence or development according to the results of existing research; (2) SNPs that may influence function or expression of PD-1 and PD-L1 by NCBI; (3) minor allele frequency (MAF) $\geq 5 \%$ in Chinese Han population.

$4 \mathrm{~mL}$ peripheral blood samples was collected in EDTA anticoagulant tubes for each patient. Genomic DNA was extracted using Genomic DNA Purification Kit according to the standard protocols. Genotyping of each polymorphism in the PD-1 and PD-L1 genes was carried out by the pyrosequencing PSQ 24 System using PyroMark Gold reagents (QIAGEN). Primers were designed using Pyrosequencing ${ }^{\mathrm{TM}}$ Assay Design Software and Primer Premier 5. The genomic region of interest was amplified by polymerase chain reaction (PCR) using one regular primer and one biotinylated primer; PCR products were sequenced using sequencing primer on
TABLE 1: Characteristics of T1DM patients and normal controls in this study.

\begin{tabular}{lccc}
\hline Characteristic & All & Type 1 diabetes & Control \\
\hline Total number & 266 & 166 & 100 \\
Gender & & & \\
$\quad$ Female $(n)$ & 146 & 82 & 64 \\
$\quad$ Male $(n)$ & 120 & 84 & 36 \\
Age (year) & $34.1 \pm 15.9$ & $25.9 \pm 12.3$ & $47.7 \pm 11.1$ \\
Diabetes duration (year) & - & $7.3 \pm 6.5$ & - \\
HbA1c (\%) & - & $8.7 \pm 2.8$ & - \\
Presence of AABs & - & 97 & - \\
(IAA, ICA, and GADA) & & & \\
\hline
\end{tabular}

Data are mean-standard deviation (SD); HbAlc: glycated hemoglobin; AABs: subjects tested for autoantibodies; IAA: insulin autoantibody; ICA: islet cell autoantibody; GADA: glutamic acid decarboxylase autoantibody.

PSQ 24 System. All the primers used in the present study were shown in Table 3.

2.3. Statistical Analysis. The chi-squared test was used to evaluate the Hardy-Weinberg equilibrium for allele frequencies of all SNPs. Unconditional logistic regression was conducted to calculate the adjusted odds ratio (OR) and 95\% confidence intervals (95\% CI). The associations between genotypes and T1DM susceptibility were tested using logistic regression analysis with Plink under additive, dominant, and recessive models. The test of significance was set at twotailed $P=0.05$.

The secondary structure of the mRNA was simulated using online webserver RNAfold (http://rna.tbi.univie.ac.at/ cgi-bin/RNAWebSuite/RNAfold.cgi), which is a recognized software providing structure prediction service [24].

\section{Results}

166 T1DM patients and 100 healthy controls were listed into the present study. All subjects were Chinese and had a mean (SD) age of $34.1 \pm 15.9$ at the time of recruitment. 8 SNPs were genotyped in these subjects, only five of which conformed to Hardy-Weinberg equilibrium $(P>0.05)$ (shown in Table 2). Thus, we took rs2227981, rs34819629, rs4143815, rs2297136, and rs2297137 into final analyses. The results were summarized in Table 4 .

There was a significant association between the PD-L1 SNP rs4143815 and T1DM susceptibility in the additive model (OR 0.50; $P=0.003$ ), dominant model (OR 0.31; $P=0.012$ ), and the recessive model (OR 0.46; $P=0.028$ ). No significant association was observed between other SNPs and T1DM susceptibility. Considering multiple-testing correction, we calculated the $P$ value for the SNPs by false detection rate (FDR) for correction. According to these results, rs4143815 remained significant in the additive model $(P=0.045)$.

In an exploratory analysis, clinical characteristics related to T1DM were compared among different genotypes of PD-L1 rs4143815 (shown in Table 5). Age, gender, HbA1c, LDL, and HDL did not vary considerably among genotypes 
TABLE 2: Eight single nucleotide polymorphisms examined in the present study.

\begin{tabular}{|c|c|c|c|c|c|c|}
\hline Gene & SNP & Chromosome & Position & Mutation & MAF & HWE \\
\hline \multirow{5}{*}{ PDCD1 } & rs34819629 & $2: 241852468$ & Intron & G/A & $\mathrm{A}=0.44$ & $P=0.170$ \\
\hline & rs11568821 & $2: 241851760$ & Intron & $\mathrm{C} / \mathrm{T}$ & $C=1.0$ & $P<0.001$ \\
\hline & rs10204525 & $2: 241850169$ & Intron & $\mathrm{C} / \mathrm{T}$ & $C=0.50$ & $P=0.002$ \\
\hline & rs2227982 & $2: 241851281$ & Intron & G/A & $A=0.60$ & $P<0.001$ \\
\hline & rs2227981 & $2: 241851121$ & Intron & $\mathrm{G} / \mathrm{T}$ & $\mathrm{T}=0.25$ & $P=0.758$ \\
\hline \multirow{3}{*}{ PD-L1 } & rs2297136 & $9: 5467955$ & $3^{\prime} \mathrm{UTR}$ & $\mathrm{A} / \mathrm{G}$ & $G=0.16$ & $P=0.197$ \\
\hline & rs4143815 & $9: 5468257$ & $3^{\prime} \mathrm{UTR}$ & $\mathrm{C} / \mathrm{G}$ & $C=0.46$ & $P=0.752$ \\
\hline & rs2297137 & 9:5465732 & Intron & $\mathrm{G} / \mathrm{A}$ & $\mathrm{A}=0.49$ & $P=0.845$ \\
\hline
\end{tabular}

SNP: single nucleotide polymorphism; MAF: minor allele frequency; HWE: Hardy-Weinberg equilibrium.

TABLE 3: All the primers used in the present study.

\begin{tabular}{|c|c|c|}
\hline rs Number & Primer & Sequence \\
\hline & $\mathrm{F}$ & GTCCTGCACCTGGGGAATG \\
\hline \multirow[t]{3}{*}{ rs34819629 } & R-Biotin & TCTGGAAGGGCACAAAGGTC \\
\hline & Sequencing primer & ACCTGGGGAATGGTG \\
\hline & $\mathrm{F}$ & CCCCAGGCAGAACCTCAAT \\
\hline \multirow[t]{3}{*}{ rs11568821 } & R-Biotin & GACCGCAGGCAGGCACATAT \\
\hline & Sequencing primer & CCCCAGCCCACCTGC \\
\hline & F-Biotin & CTGACTCССТСТСССТTTCTC \\
\hline \multirow[t]{3}{*}{ rs10204525 } & $\mathrm{R}$ & AAATCCAGCTCCCCATAGTCC \\
\hline & Sequencing primer & GAGAACACAGGCACG \\
\hline & $\mathrm{F}$ & GCAAAGGCATTCCACTGTTC \\
\hline \multirow[t]{3}{*}{ rs2297137 } & R-Biotin & ACСССТTACGCTTCATCTTCAC \\
\hline & Sequencing primer & GCATTCCACTGTTCAA \\
\hline & $\mathrm{F}$ & TTTCCAGTGGCGAGAGAAGA \\
\hline \multirow[t]{3}{*}{ rs2227981 } & R-Biotin & GGCCAAGAGCAGTGTCCA \\
\hline & Sequencing primer & CCGCCCGCAGGGGCT \\
\hline & $\mathrm{F}$ & ACGTAATCCAGCATTGGAACTT \\
\hline \multirow[t]{3}{*}{ rs2297136 } & R-Biotin & TTCAGTGCTTGGGCCTTTTAA \\
\hline & Sequencing primer & CAAGAGGAAGGAATGG \\
\hline & $\mathrm{F}$ & CTTTGCCTCCACTCAATGC \\
\hline \multirow[t]{3}{*}{ rs4143815 } & R-Biotin & TACTGTCCCGTTCCAACACTG \\
\hline & Sequencing primer & ACTCAATGCCTCAATTT \\
\hline & $\mathrm{F}$ & GGTTCGGTGCCGGTACTG \\
\hline \multirow[t]{2}{*}{ rs2227982 } & R-Biotin & GGTCTTCTCTCGCCACTGGA \\
\hline & Sequencing primer & CAAAGAAGGAGGACCC \\
\hline
\end{tabular}

of the rs4143815 SNP. However, the presence of subjects tested for autoantibodies (AABs) was higher in T1DM patients who were homozygous for the rs4143815G/G genotype compared with $\mathrm{C}$ allele carriers $(P=0.033)$.

We also simulated the secondary structure of partial PD-L1 3'UTR mRNA with different allele carrier using RNAfold (Figure 1). Different allele carrier of rs4143815 makes a great difference to the stem-loop structure of PD-L1 $3^{\prime}$ UTR, and this may account for its different binding force between miR-570 and mRNA leading to altered expression of PD-L1 protein and different susceptibility to T1DM.

\section{Discussion}

For the past few years, increasing studies had been committed to the associations with gene polymorphisms and T1DM susceptibility. The present study was made to investigate whether genetic polymorphisms in immune checkpoints could predict the onset of T1DM. In total, 266 subjects were included in the present study. With 8 selected SNPs genotyped, PD-L1 SNP rs4143815 remained significantly associated with T1DM susceptibility after FDR correction (additive model: $P=0.045$ ). No significant associations were 
TABLe 4: Association of five single nucleotide polymorphisms with type 1 diabetes susceptibility in all subjects.

\begin{tabular}{|c|c|c|c|c|}
\hline Gene & SNP & Model & OR $(95 \% \mathrm{CI})$ & $P$ value \\
\hline \multirow{6}{*}{ PD-1 } & \multirow{3}{*}{ rs2227981 } & $\mathrm{ADD}$ & $1.05(0.64 \sim 1.72)$ & 0.846 \\
\hline & & DOM & $0.98(0.52 \sim 1.84)$ & 0.941 \\
\hline & & REC & $1.45(0.41 \sim 5.12)$ & 0.563 \\
\hline & \multirow{3}{*}{ rs34819629 } & $\mathrm{ADD}$ & $0.74(0.48 \sim 1.13)$ & 0.163 \\
\hline & & DOM & $0.62(0.30 \sim 1.27)$ & 0.190 \\
\hline & & REC & $0.70(0.34 \sim 1.43)$ & 0.326 \\
\hline \multirow{9}{*}{ PD-L1 } & \multirow{3}{*}{ rs4143815 } & $\mathrm{ADD}$ & $0.50(0.31 \sim 0.79)$ & $0.003^{*}$ \\
\hline & & DOM & $0.31(0.13 \sim 0.77)$ & $0.012^{*}$ \\
\hline & & REC & $0.46(0.23 \sim 0.92)$ & $0.028^{*}$ \\
\hline & \multirow{3}{*}{ rs2297136 } & $\mathrm{ADD}$ & $0.83(0.48 \sim 1.43)$ & 0.504 \\
\hline & & DOM & $0.76(0.39 \sim 1.48)$ & 0.421 \\
\hline & & REC & $1.00(0.21 \sim 4.76)$ & 0.995 \\
\hline & \multirow{3}{*}{ rs2297137 } & $\mathrm{ADD}$ & $0.82(0.53 \sim 1.28)$ & 0.388 \\
\hline & & DOM & $0.61(0.29 \sim 1.30)$ & 0.203 \\
\hline & & REC & $0.97(0.46 \sim 2.05)$ & 0.930 \\
\hline
\end{tabular}

ADD: additive model; DOM: dominant model; REC: recessive model; additive model: comparing the minor allele with major allele subjects; dominant model: comparing carriers of the minor allele with the major homozygous subjects; recessive model: comparing carriers of the major allele with the minor homozygous subjects.

TABLE 5: Clinical characteristics of T1DM patients in different genotype of rs4143815.

\begin{tabular}{|c|c|c|c|c|}
\hline & \multicolumn{4}{|c|}{ PD-L1 rs4143815 } \\
\hline & $\mathrm{G} / \mathrm{G}(n=43)$ & $\mathrm{G} / \mathrm{C}(n=85)$ & $\mathrm{C} / \mathrm{C}(n=34)$ & $P$ value \\
\hline Age (years) & $25.7 \pm 11.6$ & $26.8 \pm 13.5$ & $24.5 \pm 10.5$ & 0.661 \\
\hline Gender ( $\%$ males) & 46.5 & 55.3 & 44.1 & 0.447 \\
\hline HbAlc (\%) & $9.0 \pm 2.5$ & $8.6 \pm 2.8$ & $8.6 \pm 2.7$ & 0.737 \\
\hline LDL & $2.7 \pm 1.2$ & $2.6 \pm 0.7$ & $2.6 \pm 0.6$ & 0.565 \\
\hline HDL & $1.5 \pm 0.5$ & $1.6 \pm 0.6$ & $1.6 \pm 0.4$ & 0.212 \\
\hline Presence of AABs (IAA, ICA, GADA) (\%) & 72.1 & 49.4 & 67.6 & $0.033^{*}$ \\
\hline
\end{tabular}

Data are mean-standard deviation (SD); HbA1c: glycated hemoglobin; LDL: low-density lipoprotein; HDL: high-density lipoprotein; AABs: subjects tested for autoantibodies; IAA: insulin autoantibody; ICA: islet cell autoantibody; GADA: glutamic acid decarboxylase autoantibody.

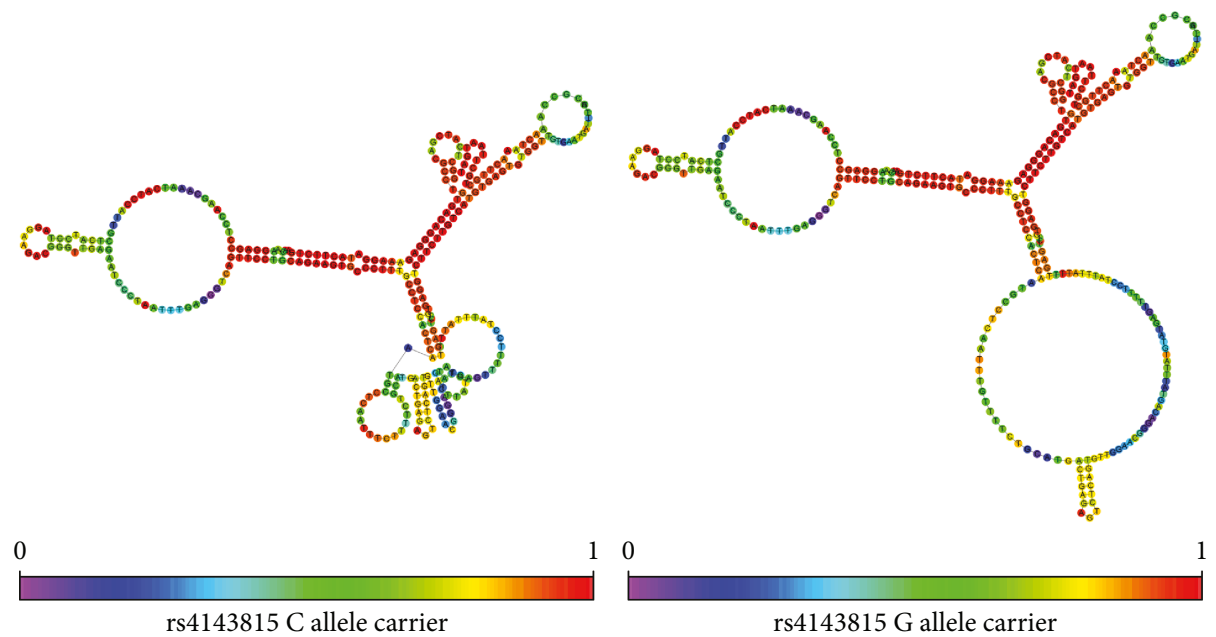

FIGURE 1: The secondary structure of partial PD-L1 3'UTR mRNA with different allele carrier of rs4143815 using RNAfold (http://rna.tbi. univie.ac.at/cgi-bin/RNAWebSuite/RNAfold.cgi). 
observed with other SNPs. Furthermore, T1DM patients homozygous for the rs $4143815 \mathrm{G} / \mathrm{G}$ genotype showed higher levels of $\mathrm{AAB}$ positive incidence compared with $\mathrm{C}$ allele carriers $(P=0.033)$.

PD-1 is a core costimulatory molecule expressed by $\mathrm{T}$ cells that interact with its ligands PD-L1 and PD-L2 on antigen-presenting cells. PD-L1 was shown to be overexpressed in human gastrointestinal tumors [8]. PD-L1 negatively regulated immune response by interacting with PD-1 on $\mathrm{T}$ cells and therefore promoting $\mathrm{T}$ cell apoptosis [25]. Anti-PD-1 antibody treatment has been applied to the treatment of numerous advanced cancers; however, insulindependent diabetes had been reported during the anti-PD-1 immunotherapy process in both mouse models and human cases [11-13].

Increasing studies had been committed to the association with PD-1/PD-L1 and type 1 diabetes; low serum level of PD-1 and PD-L1 was reported being associated with T1DM in Japanese and Chilean patients [3, 21]. Association of polymorphisms of the PD-1and PD-L1 with T1DM has been evaluated previously in other countries [21-23]. Nevertheless, the genetic background of type 1 diabetes differs greatly among the different population.

In the present study, rs4143815 was found being associated with T1DM susceptibility. People carrying the $\mathrm{C}$ allele of rs4143815 suffering less risk of T1DM and T1DM patients with $G / G$ genotype showed higher levels of $A A B$ positive incidence compared with $\mathrm{C}$ allele carriers. Since the level of AABs has high associations of T1DM, the results of AABs reinforce the importance of rs4143815. People carrying the $\mathrm{C}$ allele of rs4143815 suffer lower $\mathrm{AAB}$ level and less risk of T1DM. However, HbA1c did not vary considerably among genotypes of the rs 4143815 $\mathrm{SNP}$. Since HbA1c is an important indicator for glycemic control and has little relevance to the susceptibility of the disease, this may be induced by the different treatment stage of T1DM patients.

It had been reported that rs4143815 was associated with the increased risk of gastric cancer [26, 27] and non-small-cell lung cancer [28, 29], possibly resulted from the suppression of immunological tumor surveillance by increased PD-L1 expression [30]. rs4143815 was also found being associated with the risk of T1DM in Chile [21]. This previous study comprised 205 T1DM patients and 205 normal children; however, the HWE equilibrium of rs4143815 for T1DM was 0.006 . Our results use pyrosequencing to genotype all the SNPs, and the HWE equilibrium of rs4143815 is 0.752 . With proper HWE equilibrium and advanced genotyping method, our results reinforce the importance of rs4143815 in T1DM susceptibility in Chinese population.

The rs4143815 is located in the $3^{\prime}$ UTR of PD-L1 and binds to miR-570 [26]. Different allele may change the secondary structure of PD-L1 $3^{\prime}$ UTR. RNAfold was used to predict the secondary structure of PD-L1 $3^{\prime}$ UTR; G allele carrier shows a bigger stem-loop structure compared with $\mathrm{C}$ allele (shown in Figure 1) which may increase the interaction between PD-L1 3'UTR and miR-570 leading to lower PDL1 expression. Lee et al. found that rs4143815 G allele exhibited a decreased transcription activity compared with $\mathrm{C}$ allele though luciferase report [31] which accords with our previous prediction.

Several studies had been committed to the association with PD-1 and autoimmune disease. PD 1.2, PD1.3, PD1.5, and PD1.9 were found being associated with systemic lupus erythematosus, ankylosing spondylitis, and allergic bronchial asthma [14-17]. However, our results showed that there are no associations between PD-1 polymorphisms and T1DM susceptibility. This may be due to different pathogenesis between T1DM and other autoimmune diseases.

Our study has some limitations. With limited SNPs and small sample size, the final result may have a certain deviation. We will select more typical SNPs and increase the sample size in the next step, and we will use gene chip and conduct multicenter studies to revalidate our results in the future.

\section{Conclusion}

In this study, our results indicate that rs4143815 of PD-L1 is significantly associated with T1DM susceptibility. Importantly, this SNP remained significantly after FDR correction. Furthermore, the presence of AABs is higher in T1DM patients homozygous for the rs $4143815 \mathrm{G} / \mathrm{G}$ genotype compared with $\mathrm{C}$ allele carriers.

\section{Data Availability}

The data used to support the findings of this study are available from the corresponding author upon request.

\section{Ethical Approval}

All procedures followed were in accordance with the ethical standards of the responsible committee on human experimentation (institutional and national) and with the Helsinki Declaration of 1975, as revised in 2008.

\section{Conflicts of Interest}

The authors declare that they have no conflict of interest.

\section{Authors' Contributions}

Chenyue Qian and Heming Guo contributed equally to this work.

\section{Acknowledgments}

This study was funded by grants from the National Natural Science Foundation of China (Grant no. 81471041 to Ji Hu, 81600607 to Chen Fang, and 81502865 to Yun Huang). This work was also supported by the Open Research Project of Shanghai Key Laboratory of Diabetes Mellitus (SHKLDKF-1604) and Ministry of Science and Technology of China under Award Number 2016YFC1305202. This work was also supported by Second Affiliated Hospital of Soochow University Science Foundation (no. SDFEYQN1604) and Suzhou Science Foundation (no. SYSD2016148). 


\section{References}

[1] J. Tian, Y. Liu, Y. Liu, K. Chen, and S. Lyu, "Cellular and molecular mechanisms of diabetic atherosclerosis: herbal medicines as a potential therapeutic approach," Oxidative Medicine and Cellular Longevity, vol. 2017, Article ID 9080869, 16 pages, 2017.

[2] B. T. Fife and J. A. Bluestone, "Control of peripheral T-cell tolerance and autoimmunity via the CTLA- 4 and PD- 1 pathways," Immunological Reviews, vol. 224, no. 1, pp. 166-182, 2008.

[3] R. Fujisawa, F. Haseda, C. Tsutsumi et al., "Low programmed cell death-1 (PD-1) expression in peripheral CD4 ${ }^{+} \mathrm{T}$ cells in Japanese patients with autoimmune type 1 diabetes," Clinical \& Experimental Immunology, vol. 180, no. 3, pp. 452-457, 2015.

[4] E. Nolan, P. Savas, A. N. Policheni et al., "Combined immune checkpoint blockade as a therapeutic strategy for BRCA1mutated breast cancer," Science Translational Medicine, vol. 9, no. 393, article eaal4922, 2017.

[5] A. Bang, T. J. Wilhite, L. R. G. Pike et al., "Multicenter evaluation of the tolerability of combined treatment with PD-1 and CTLA-4 immune checkpoint inhibitors and palliative radiation therapy," International Journal of Radiation Oncology, Biology, Physics, vol. 98, no. 2, pp. 344-351, 2017.

[6] S. Marwitz, S. Scheufele, S. Perner, M. Reck, O. Ammerpohl, and T. Goldmann, "Epigenetic modifications of the immunecheckpoint genes CTLA4 and PDCD1 in non-small cell lung cancer results in increased expression," Clinical Epigenetics, vol. 9, no. 1, p. 51, 2017.

[7] C. Wu, Y. Zhu, J. Jiang, J. Zhao, X. G. Zhang, and N. Xu, "Immunohistochemical localization of programmed death-1 ligand-1 (PD-L1) in gastric carcinoma and its clinical significance," Acta Histochemica, vol. 108, no. 1, pp. 19-24, 2006.

[8] W. Zou and L. Chen, "Inhibitory B7-family molecules in the tumour microenvironment," Nature Reviews Immunology, vol. 8, no. 6, pp. 467-477, 2008.

[9] Q. Li, J. F. Gao, and B. L. Qi, "PDCD1 strengthens the sensitivity of ovarian cancer to cisplatin chemotherapy by promoting apoptosis," Journal of Balkan Union of Oncology, vol. 22, no. 3, pp. 746-756, 2017.

[10] J. Y. Kim, E. Lee, K. Park et al., "Immune signature of metastatic breast cancer: identifying predictive markers of immunotherapy response," Oncotarget, vol. 8, no. 29, pp. 47400-47411, 2017.

[11] M. J. I. Ansari, A. D. Salama, T. Chitnis et al., "The programmed death-1 (PD-1) pathway regulates autoimmune diabetes in nonobese diabetic (NOD) mice," Journal of Experimental Medicine, vol. 198, no. 1, pp. 63-69, 2003.

[12] J. Hughes, N. Vudattu, M. Sznol et al., "Precipitation of autoimmune diabetes with anti-PD-1 immunotherapy," Diabetes Care, vol. 38, no. 4, pp. e55-e57, 2015.

[13] J. Martin-Liberal, A. J. S. Furness, K. Joshi, K. S. Peggs, S. A. Quezada, and J. Larkin, "Anti-programmed cell death-1 therapy and insulin-dependent diabetes: a case report," Cancer Immunology, Immunotherapy, vol. 64, no. 6, pp. 765-767, 2015.

[14] S. Dai, R. Jia, X. Zhang, Q. Fang, and L. Huang, "The PD-1/ PD-Ls pathway and autoimmune diseases," Cellular Immunology, vol. 290, no. 1, pp. 72-79, 2014.

[15] E. V. Dmitrieva-Zdorova, M. V. Gabaeva, Y. A. Seregin, N. V. Bodoev, and O. E. Voronko, "PDCD1 PD-1.3 polymorphism and allergic bronchial asthma in Russian and Buryat patients," Journal of Asthma, vol. 54, no. 1, pp. 46-52, 2017.

[16] K. Chua, L. Lian, X. Sim, T. Cheah, and T. Lau, "Association between PDCD1 gene polymorphisms and risk of systemic lupus erythematosus in three main ethnic groups of the Malaysian population," International Journal of Molecular Sciences, vol. 16, no. 12, pp. 9794-9803, 2015.

[17] M. Yang, Y. Zou, Y. Bai, and M. Li, “The programmed cell death 1 gene polymorphisms (PD $1.3 \mathrm{G} / \mathrm{A}, \mathrm{PD} 1.5 \mathrm{C} / \mathrm{T}$ and PD $1.9 \mathrm{C} / \mathrm{T}$ ) and susceptibility to ankylosing spondylitis: a meta-analysis," Journal of Orthopaedic Science, vol. 20, no. 1, pp. 55-63, 2015.

[18] B. Zhang, Y. Li, X. Zhong, W. Huang, L. Nie, and W. Zhang, "Establishment of retinoblastoma model in NOD-SCID mice and study of metastasis," Yan Ke Xue Bao, vol. 21, no. 3, pp. 185-191, 2005.

[19] J. Wang, T. Yoshida, F. Nakaki, H. Hiai, T. Okazaki, and T. Honjo, "Establishment of NOD-Pdcd1 ${ }^{-/-}$mice as an efficient animal model of type I diabetes," Proceedings of the National Academy of Sciences of the United States of America, vol. 102, no. 33, pp. 11823-11828, 2005.

[20] M. L. Colli, J. L. E. Hill, L. Marroquí et al., "PDL1 is expressed in the islets of people with type 1 diabetes and is up-regulated by interferons- $\alpha$ and $-\gamma$ via IRF1 induction," EBioMedicine, vol. 36, pp. 367-375, 2018.

[21] C. Pizarro, D. F. García-Díaz, E. Codner, F. Salas-Pérez, E. Carrasco, and F. Pérez-Bravo, "PD-L1 gene polymorphisms and low serum level of PD-L1 protein are associated to type 1 diabetes in Chile," Diabetes/Metabolism Research and Reviews, vol. 30, no. 8, pp. 761-766, 2014.

[22] Y. H. Lee, S. C. Bae, J. H. Kim, and G. G. Song, "Meta-analysis of genetic polymorphisms in programmed cell death 1 . Associations with rheumatoid arthritis, ankylosing spondylitis, and type 1 diabetes susceptibility," Zeitschrift für Rheumatologie, vol. 74, no. 3, pp. 230-239, 2015.

[23] Y. Hiromine, H. Ikegami, T. Fujisawa et al., "Trinucleotide repeats of programmed cell death-1 gene are associated with susceptibility to type 1 diabetes mellitus," Metabolism, vol. 56, no. 7, pp. 905-909, 2007.

[24] R. B. Denman, "Using RNAFOLD to predict the activity of small catalytic RNAs," BioTechniques, vol. 15, no. 6, pp. 1090-1095, 1993.

[25] H. Dong, S. E. Strome, D. R. Salomao et al., "Tumor-associated B7-H1 promotes T-cell apoptosis: a potential mechanism of immune evasion," Nature Medicine, vol. 8, no. 8, pp. 793-800, 2002.

[26] W. Wang, F. Li, Y. Mao et al., "A miR-570 binding site polymorphism in the $B 7-H 1$ gene is associated with the risk of gastric adenocarcinoma," Human Genetics, vol. 132, no. 6, pp. 641-648, 2013.

[27] L. H. Tao, X. R. Zhou, F. C. Li et al., "A polymorphism in the promoter region of PD-L1 serves as a binding-site for SP1 and is associated with PD-L1 overexpression and increased occurrence of gastric cancer," Cancer Immunology, Immunotherapy, vol. 66, no. 3, pp. 309-318, 2017.

[28] W. Du, J. Zhu, Y. Chen et al., "Variant SNPs at the microRNA complementary site in the $\mathrm{B} 7-\mathrm{H} 13^{\prime}$-untranslated region increase the risk of non-small cell lung cancer," Molecular Medicine Reports, vol. 16, no. 3, pp. 2682-2690, 2017.

[29] T. Nomizo, H. Ozasa, T. Tsuji et al., "Clinical impact of single nucleotide polymorphism in PD-L1 on response to nivolumab 
for advanced non-small-cell lung cancer patients," Scientific Reports, vol. 7, no. 1, article 45124, 2017.

[30] X. L. Shi, S. Mancham, B. E. Hansen et al., "Counter-regulation of rejection activity against human liver grafts by donor PD-L1 and recipient PD-1 interaction," Journal of Hepatology, vol. 64, no. 6, pp. 1274-1282, 2016.

[31] S. Y. Lee, D. K. Jung, J. E. Choi et al., "Functional polymorphisms in $P D-L 1$ gene are associated with the prognosis of patients with early stage non-small cell lung cancer," Gene, vol. 599, pp. 28-35, 2017. 


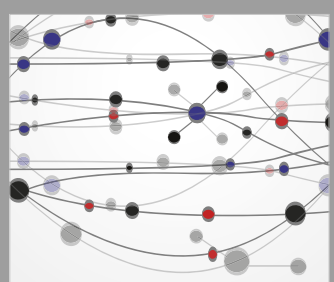

The Scientific World Journal
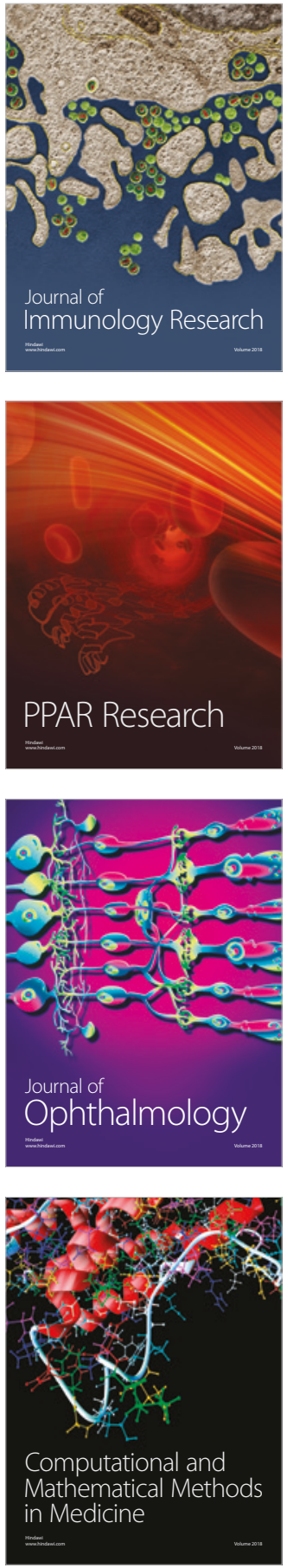

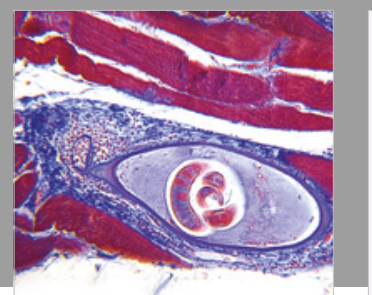

Gastroenterology Research and Practice

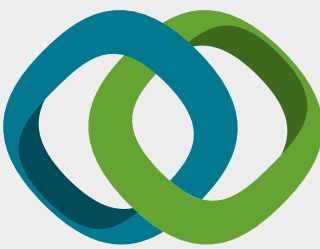

\section{Hindawi}

Submit your manuscripts at

www.hindawi.com
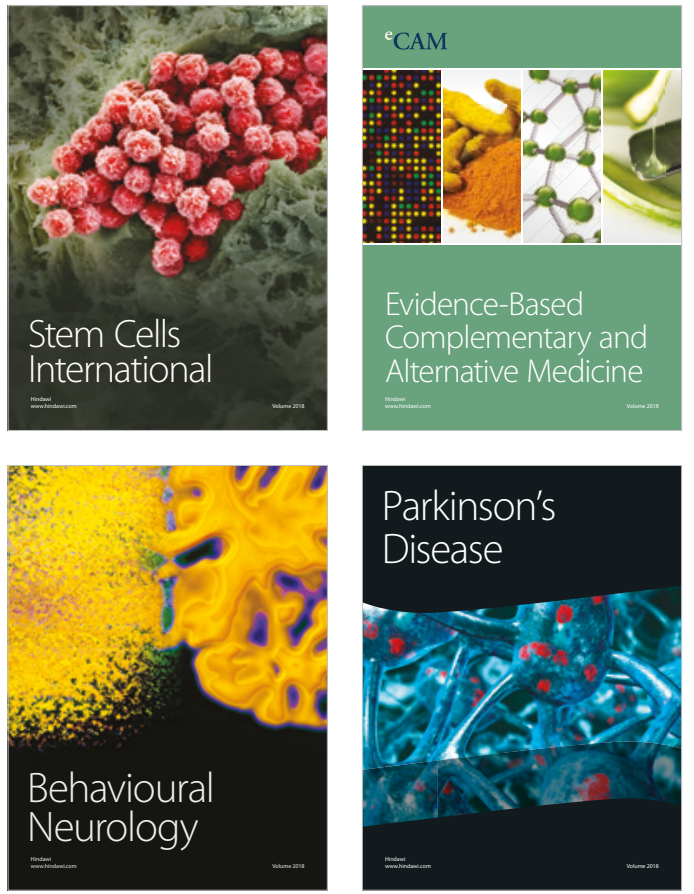

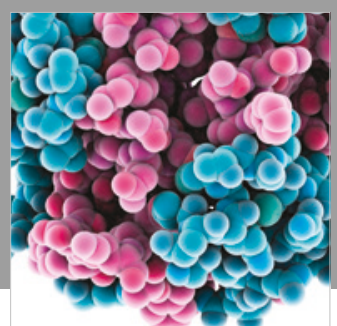

ournal of

Diabetes Research

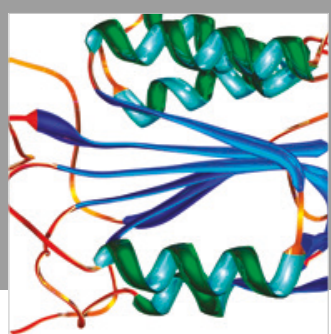

Disease Markers
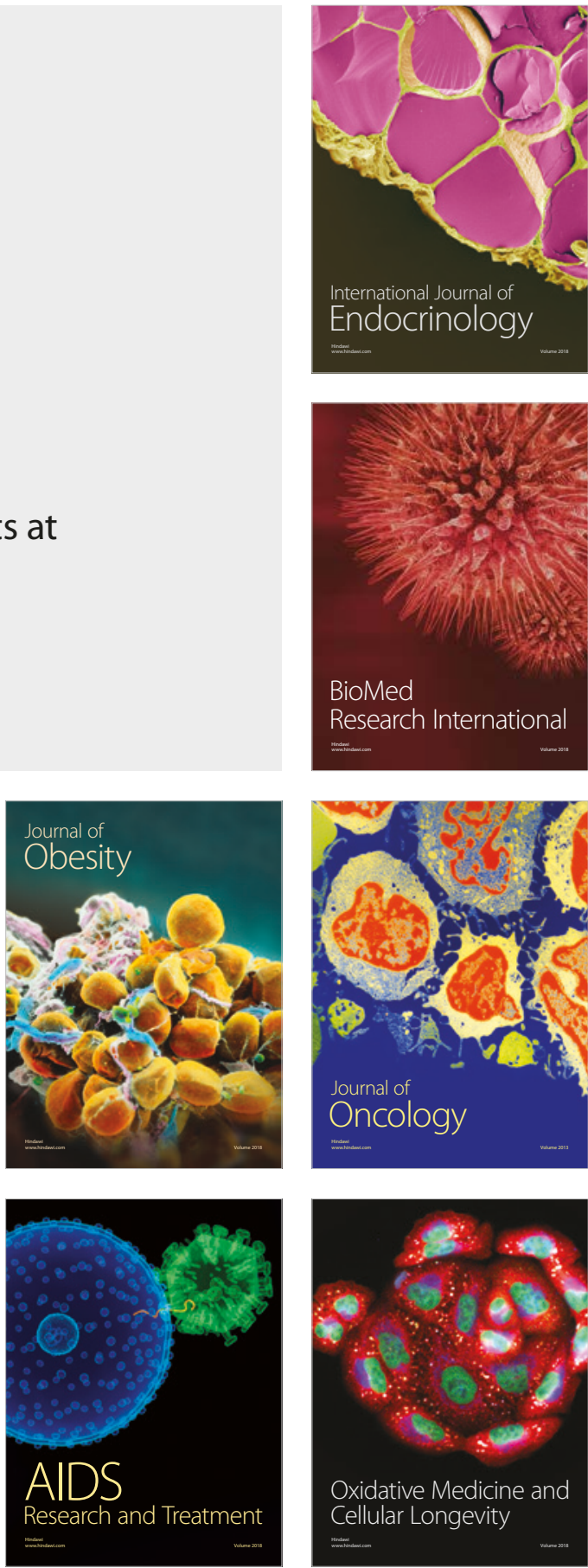